\title{
Cosmic Ray and Geomagnetic Response to High Speed Solar Wind Streams
}

\author{
N.K.Sharma \& Tufail Ahmad* \\ Faculty of Technology, C.S.A University of Agriculture \& Technology Campus-Etawah (U.P.) India -206001 \\ * University Polytechnic, Aligarh Muslim University, Aligarh
}

\begin{abstract}
The study of cosmic ray intensity and geomagnetic response have been carried out with the data registered by ground based and satellite data on 24-hour scale during 1996, 2000 and 2008 which corresponds to solar minimum of solar activity cycle 23 during 1996 and 2008 followed by a solar maximum in the year of 2000. The minimum of solar activity cycles are characterized by different polarity of the north pole of the sun's magnetic field as the polarity reversal occurs during 2000. The results presented in this paper indicate that cosmic ray intensity and geomagnetic field is highly disturbed during the passage of these streams at Earth. The average time-profile of the decrease in cosmic ray intensity produced by streams associated with coronal holes and solar flares during the maximum epoch of solar activity cycle 23 is explained in terms of existing models of solar modulation of cosmic rays. The comparison of modulation features during the different magnetic conditions i.e. during 1996 \& 2008 shows that drift effects are less dominant in the short-term modulation of cosmic rays. These results indicate that the solar wind streams are playing an important role in the transient modulation of cosmic ray intensity.
\end{abstract}

Key Words: Sun: Solar activity, Polarity Reversal, Galactic Cosmic rays (GCR) and Forbush decreases (Fds)

\section{Introduction}

It is well known that temporal variations in the solar wind streams at the earth's location (1AU) exert a strong influence on the heliosphere, galactic cosmic rays and terrestrial magnetosphere. The continuous measurements of the interplanetary medium and the Sun have produced exciting and important changes in our knowledge of interplanetary phenomena. One of the most important phenomena of the solar terrestrial physics is the occurring streams in the solar wind near the Earth. Streams are quasi-stationary, hot and low density flows originating in coronal holes of the Sun. The satellite data collected for various solar cycles have shown that high and low-speed streams are continuously reaching the earth and they have different origins. Two types of solar wind streams have been identified as regular and recurrent streams coming from coronal holes and complex and transient streams associated with solar flares. The origin of the low speed solar wind strems is still an open problem (Iucci et al. 1979). The study of the relationship between interplanetary plasma parameters and geomagnetic index has been a common topic of investigation since the beginning of the space era. Furthermore theoretical work on the physical meaning of the functional relationship between interplanetary and geomagnetic parameters is continuously in progress and models are developed to explain the energy transfer from solar wind to the magnetosphere (Crooker and Siscoe, 1986).

Short-term decrease in cosmic ray intensity observed by ground-based detectors are, in general broadly classified into two categories: (1) The classical (generally non-recurrent) Forbush decreases associated with transient on the Sun and (ii) the generally recurrent decreases associated with co rotating high speed solar wind streams from coronal holes (Venkatesan et al., 1978; Vershell et al., 1975 and Shah et al., 1978). Space craft such as Voyagers and Pioneers have also observed some of these decreases to various distances from the Sun, with delays from those observed at $1 \mathrm{AU}$, depending on the solar wind speed.

The intent of this work is to study the cosmic ray and geomagnetic response to high speed solar wind streams associated with coronal holes and solar flares during the minimum and maximum epoch of solar activity cycle 23. The aim of this analysis is to study the effect of large scale magnetic field polarity on the average time profile of cosmic ray decreases along with the role of high speed solar wind streams (HSS) and associated disturbances on the short term modulation of cosmic rays.

\section{Data And Analysis}

Cosmic ray measurements in Oulu (65.05 N, 25.47 E) started in 1964 with standard 9-NM-64 neutron monitor. The local vertical geomagnetic cut off rigidity is about $0.8 \mathrm{GV}$. The data of the Oulu neutron monitor Is used in the analysis because this neutron monitor is one of the most stable and reliable station of the world neutron Monitor Network the website used to access the cosmic ray and geomagnetic data (http://www.cosmicrays.oulu.fi) is used in the analysis. 
The present analysis deals with the classification of streams into two group according to their time duration as well as per their association with coronal holes and solar flares (i) Short Streams or S-streams of time duration $\Delta \mathrm{t} \leq 5$ days (ii) Medium streams or M-streams of time duration $5 \leq \Delta \mathrm{t} \geq 9$ days during the minimum and maximum of solar activity cycle 23 i.e. (1996, 2000 and 2008) are considered in the analysis. The Chree-method was applied by using the daily average value of cosmic ray intensity and the daily values of geomagnetic index which are used as an index parameter to represent the geomagnetic field variation.

\section{Results And Discussion}

The Chree analysis of superposed epoch has been adopted to study the effect of short and medium high speed solar wind streams associated with coronal holes and solar flares on cosmic ray intensity using the daily average cosmic ray intensity of Oulu neutron monitor during minimum and maximum of solar activity cycle 23. To study the influence of short duration streams on cosmic ray intensity and geomagnetic index, Chree analysis of superposed epoch for -3 to +5 days and for medium class high speed solar wind streams the epoch for -3 to +9 days is considered and plotted in Figures as a percent deviation of cosmic ray intensity and Ap index during 1996, 2000 and 2008. Deviation for each event is obtained from the overall average of 9 and 13 days for short and medium streams respectively.

As depicted in Fig.1(a) the cosmic ray intensity decrease starts at -1 day and reaches to a maximum decrease on +2 day for short streams also a phenomena of over-recovery (i.e. the return of cosmic ray counts to a value higher than the pre-decrease level) is observed for these streams during 1996, which corresponds to the outward polarity of the sun's magnetic field, the occurrence rate of streams associated with solar flares is insignificant during 1996 solar minimum, the analyzed streams along with their association with coronal holes and solar flares during 1996, 2000 and 2008 are summarized in Table 1. Fig.1 (b) depicts the superposed epoch plot of cosmic ray intensity for medium high speed solar wind streams associated with coronal holes during 1996. The cosmic ray intensity starts decrease after the arrival of streams at Earth and reaches to a maximum decrease on +1 day and then recovery starts gradually to its pre-decrease level here again phenomena of over recovery is observed for these streams. The average time profile of cosmic ray intensity seems to be of Forbush decrease type (i.e. fast decrease and slow recovery) during medium class high speed solar wind streams during 1996.

As depicted in Fig.2 (a) the cosmic ray intensity decrease starts after the arrival of short streams associated with coronal holes and solar flares. The cosmic ray intensity reaches to maximum decrease on +2 day for streams associated with coronal holes than recovery starts gradually, while for streams associated with solar flares the decrease reaches to maximum on +1 day and then recovery starts gradually, the average time profile in this case is of Forbush decrease type. Also a two step-decrease is observed for short streams associated with solar flares during 2000 which corresponds to the maximum of solar activity cycle 23. Fig.2 (b) depicts the superposed epoch plots of cosmic ray intensity for medium high speed solar wind streams during 2000.The average time profile of cosmic ray intensity of medium high speed solar wind streams associated with coronal holes and solar flares shows the decrease in cosmic ray intensity after the arrival of these streams on Earth but the magnitude of the decrease is large for the streams associated with solar flares and a phenomena of overrecovery is also associated for the medium class streams associated with coronal holes and solar flares (Fig.2b).

Table -1 Statistics of the events analyzed

\begin{tabular}{|c|c|c|c|c|}
\hline Types of the stream & $\begin{array}{c}1996 \text { (minimum of solar } \\
\text { cycle 23) } \\
\text { CH Type }\end{array}$ & \multicolumn{2}{|c|}{ 2000 (max. of solar cycle 23) } & \multirow{2}{*}{$\begin{array}{c}\text { 2008(minimum of } \\
\text { solar cycle 23) } \\
\text { CH Type }\end{array}$} \\
\cline { 3 - 4 } & 22 & CH Type & SF Type & \\
\hline Short Streams $\Delta \mathrm{T} \leq 5$ days & 22 & 13 & 14 & 03 \\
\hline $\begin{array}{c}\text { Medium Streams } 5 \leq \Delta \mathrm{T} \leq 9 \\
\text { days }\end{array}$ & 10 & 07 & 23 \\
\hline
\end{tabular}

As depicted in Fig.3(a) the cosmic ray intensity has a decreasing trend after the arrival of these streams on Earth but the average time profile is not very straight forward for short high speed solar wind streams during 2008 , which corresponds to inward polarity of the sun's magnetic field as the polarity reversal occurs during 2000 which corresponds to the maximum of solar activity cycle 23 , the phenomena of over-recovery is observed for these streams (Fig.3a). The magnitude of decrease of short high speed solar wind streams during 2008 is very small as compared to the magnitude of the decrease noted in the year 1996. The superposed epoch plot of cosmic ray intensity of medium class high speed solar wind streams associated with coronal holes during 2008 (Fig.3b) indicates that the magnitude of decrease in cosmic ray intensity is large as compared with the magnitude of the decrease observed in 1996 (Fig.2b). Our results presented here clearly indicate that both type of high speed solar wind streams considered in the analysis perturbed the cosmic ray intensity during the passage from the Earth. Our observational results are summarized in Table2. The results presented here indicates that the recovery time for short duration streams for two different magnetic conditions i.e. during 1996 
and 2008 is not same for short duration streams while the recovery time is same for medium stream for different magnetic conditions also the amplitudes of decrease is almost same during 1996 and 2008 which corresponds to the different polarity of the sun' magnetic field. In 2008, the recovery time is smaller for short duration streams as compared to the streams considered during 1996 (Table2). In 1996 when the northern hemisphere interplanetary magnetic field (IMF) pointed away from the sun, cosmic rays drift towards the earth from over the solar poles, and under such circumstances, the cavity left behind by propagating disturbance in equatorial region will be filled as a faster rate and consequently the recovery time will be smaller in this polarity state. As the polarity of the sun's magnetic field changes in 2000 during the maximum of solar activity cycle 23 consequently, for 2008 solar minimum the polarity of the sun's magnetic field becomes inward, under such condition particle drift towards earth from the equatorial region and drifting particles will primarily encounter the disturbance head on and the filling process is retarded in this situation. This is the consequence of driftdominated models. A number of workers have studied the role of drift on long-term modulation (e.g., Sharma and Yadav, 1993, Venkatesan and Badruddin, 1990, Potgieter, 1998 and references therein) and many of them emphasized for the dominant role of gradient and curvature drifts. On the other hand, the role of drift in the phenomenon of Forbush decrease has been studied by the limited workers (e.g., Lockwood et al 1986; Mulder and Moraal; 1986; Rana et al. 1996) and experimental results are inconclusive as regards the role of drifts during this phenomenon. Le Roux and Potgieter (1991) simulated Forbush decreases by assuming that turbulent field regions of enhanced scattering cause them and drift effects are diminished in the region that originate at the sun and propagate outwards. Our results presented here again enhance the findings of earlier investigators of view that it is difficult to establish quantitatively the role of gradient and curvature drifts in short term variations in cosmic ray intensity produced by the streams associated with coronal holes and solar flares. The results presented here also enhance the importance of the phenomena of over-recovery as reported earlier by Dumbovic et al. 2012.

Table-2 Characteristics of Modulation during minimum and maximum epochs of solar active cycle 23.

\begin{tabular}{|c|l|c|c|c|c|l|l|}
\hline Year & \multicolumn{1}{|c|}{$\begin{array}{c}\text { Class of } \\
\text { Stream }\end{array}$} & $\begin{array}{c}\text { Time of } \\
\text { decrease } \\
\text { (days) }\end{array}$ & $\begin{array}{c}\text { Time of } \\
\text { recovery } \\
\text { (days) }\end{array}$ & $\begin{array}{c}\text { Total Time } \\
\text { (days) }\end{array}$ & $\begin{array}{c}\text { Maximum } \\
\text { Decrease in } \\
\text { cosmic ray } \\
\text { intensity (- } \Delta l \%)\end{array}$ & $\begin{array}{c}\text { Nature of Time } \\
\text { Profile }\end{array}$ & $\begin{array}{c}\text { Type of } \\
\text { streams }\end{array}$ \\
\hline 1996 & S-streams & 2 & 3 & 5 & 0.12 & Gradual & CH type \\
& M-streams & 1 & 4 & 5 & 0.12 & Fds type & CH type \\
\hline 2000 & S-streams & 2 & 3 & 5 & 0.10 & Gradual & CH type \\
& S-streams & 1 & 2 & 3 & 0.50 & Fds type & SF type \\
& M-streams & 2 & 2 & 4 & 0.20 & Gradual & CH type \\
& M-streams & 4 & 4 & 8 & 2.00 & Gradual & SF type \\
\hline 2008 & S-streams & 1 & 1 & 2 & 0.01 & Not defined & CH type \\
& M-streams & 4 & 4 & 8 & 0.80 & Gradual & CH type \\
\hline
\end{tabular}

For each class of streams a superposed epoch analysis was performed, with the epochs for S-events ranging from -3 to +6 days and for M-events between -3 to +9 days on daily values of Ap index and plotted in Figs 1c \& $\mathrm{d}$ as a percent deviation of Ap index data during 1996 for short and medium duration streams. Deviation for each event is obtained from the overall average of 9 days for S-streams and 13 days for medium streams. As depicted in Fig.1 (c \& d) a sharp increase is noted on +1 day after the arrival of these streams on Earth. The average time profile of Ap index for medium high speed solar wind streams in 1996 is associated with tow-peak of different magnitude i.e. on +1 day and another peak is on +4 day after the arrival of these streams on earth. Fig $2 c$ \&d depicts the average time profile of Ap index during the maximum of solar cycle, 23 in 2000, again a sharp increase peak is observed on +1 day after the arrival of short and medium duration streams on earth. A superposed epoch plot of Ap index for short and medium duration streams during the minimum in 2008 of solar activity cycle 23 again shows a increasing peak on +1 day after the arrival of these streams on earth Fig. $3 \mathrm{c} \& \mathrm{~d}$. The results presented here further enhance the importance of various physical processes which are responsible for the short term modulation of cosmic rays. It is difficult to establish quantitatively the role of gradient and curvature drifts on average time profile of cosmic ray intensity observed during short and medium class high speed solar wind streams associated with coronal holes and solar flares because various solar activity parameters, such as solar flares, coronal holes, coronal mass ejections and current sheet tilt are supposed to be responsible for short term variations in cosmic ray intensity in general and during Forbush decreases (Fds) in particular. Many of them may occur simultaneously. Moreover, the operation of various mechanisms such as, convection, diffusion, dynamic sweeping of cosmic ray particle out of the inner heliosphere by moving compression wave, and adiabatic cooling of cosmic ray particles further complicate the problem. However, the difference observed in the morphology of average time profile of cosmic ray intensity during two periods (1996 and 2008 the minimum of solar activity cycle 23) of opposite solar polar magnetic field polarities may qualitatively be explained on the basis of drift theory. 


\section{Conclusions}

The conclusions which emerge from the present study are as follows:

1. During the minimum of solar activity cycle 23 (in 1996 \& 2008) the short duration streams associated with solar flares are insignificant in number.

2. During the maximum epoch of solar activity cycle 23 (i.e. in 2000) both type of the streams associated with coronal holes and solar flares are statistically significant in number.

3. The cosmic ray response is more effective for the streams associated with the solar flare as compared with the streams associated with the coronal holes during the maximum epoch of solar activity cycle 23 (i.e.in 2000 ).

4. No significant difference is observed in the average time profile of cosmic ray intensity during the minimum epochs of solar activity cycle 23 which corresponds to the different polarity of the sun's magnetic field.

5. The results are consistent with the model calculation based on the assumption that cosmic ray decreases are caused by turbulent field regions of enhanced scattering (i.e. the streams associated with solar flares) and diminished drifts.

6. Short and medium duration streams perturbed the geomagnetic field on day to day basis and the peak of increase coincide on +1 day for each class of streams during minimum and maximum epochs of solar activity cycle 23 .

\section{Acknowledgement}

The Authors are indebted to various experimental groups for providing the cosmic ray, solar wind streams and geophysical data. One of the author (NKS) is thankful to Dr. J.P.Yadav, of Technology, Etawah and Prof. Munna Singh, Vice-Chancellor, Chandra Dean Faculty Shekhar Azad University of Agriculture \& Technology, Kanpur for their constant encouragement during the preparation of this manuscript.

\section{References}

[1]. Crooker, N.U and Siscoe, G I: Physics of the Sun, 3P.A. Sturrock (ed.), D.Reidel Publication. Co. Dordrecht, 193-249 (1986).

[2]. Dumbovic M, Vrsnak,B , Calogovic J and Zupan R: Astronomy and Astrophysics, A28, 538 (2012).

[3]. Iucci N M, Parrisi M, storini M and Villoresi G: Nuovo Cimento, 2C, 421 (1979).

[4]. Lockwood, J.A., Webber,W R and Jokippii,J.R : J.Geophys.Res. 91, 2851 (1986).

[5]. Le Roux, J A and Potgieter M S: Astrophys Journal, 243, 531 (1991)

[6]. Mulder,M S and Morral,H: Astrophys.J. 303, L75 (1986).

[7]. Potgieter, M.S. Mrringa N., Ferreira, S.E.S, Manuel, R. and Ndiitwani, D.C.: Journal of Advance Research, 43,259 (2013).

[8]. Rana,D S, Sharma N K and Yadav R S: Solar Physics, 167,371-380 (1996).

[9]. Sharma, N.K. Yadav. R.S.: India Journal of Radio and Space Physics, 23, 165 (1994).

[10]. Shah, G.N., Kaul, C.L. Razdan, H., and Bemalkhedkar, M.M.: J. Geophys. Res. 83, 3740 (1978).

[11]. Venkatesan D and Badruddin: Space Science Review: 52, 121-194, (1990).

[12]. Venkatesan D. Shukla, A.K. and Agrawal, S.P.: Solar Physics, 81, 375 (1982).

[13]. Vershell, H.J., Mendall, R.B. Korff, S.A. and Roelof, E.C.: J. Geophys. Res. 80, 1189 (1975).

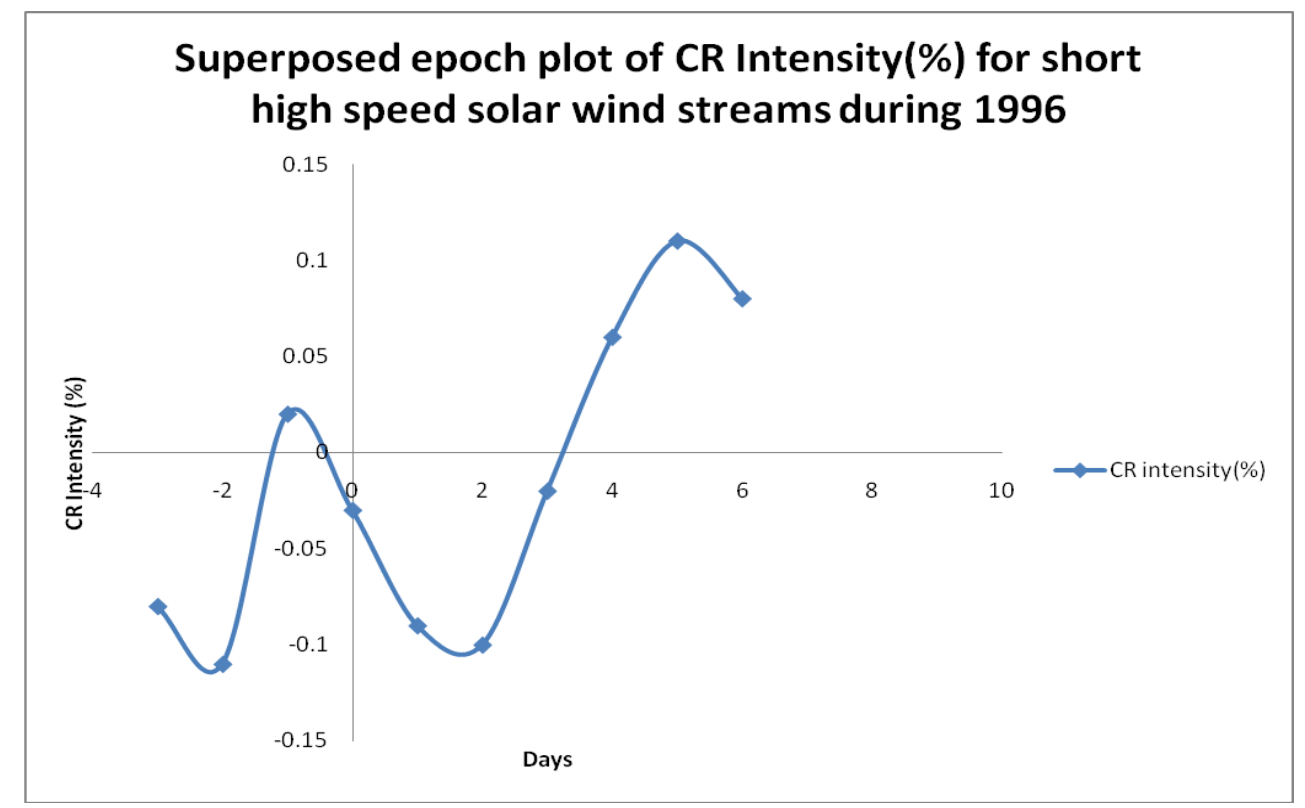

Fig 1(a): Superposed epoch plot of Oulu CR Intensity (\%) for short high speed solar wind streams during 1996. 


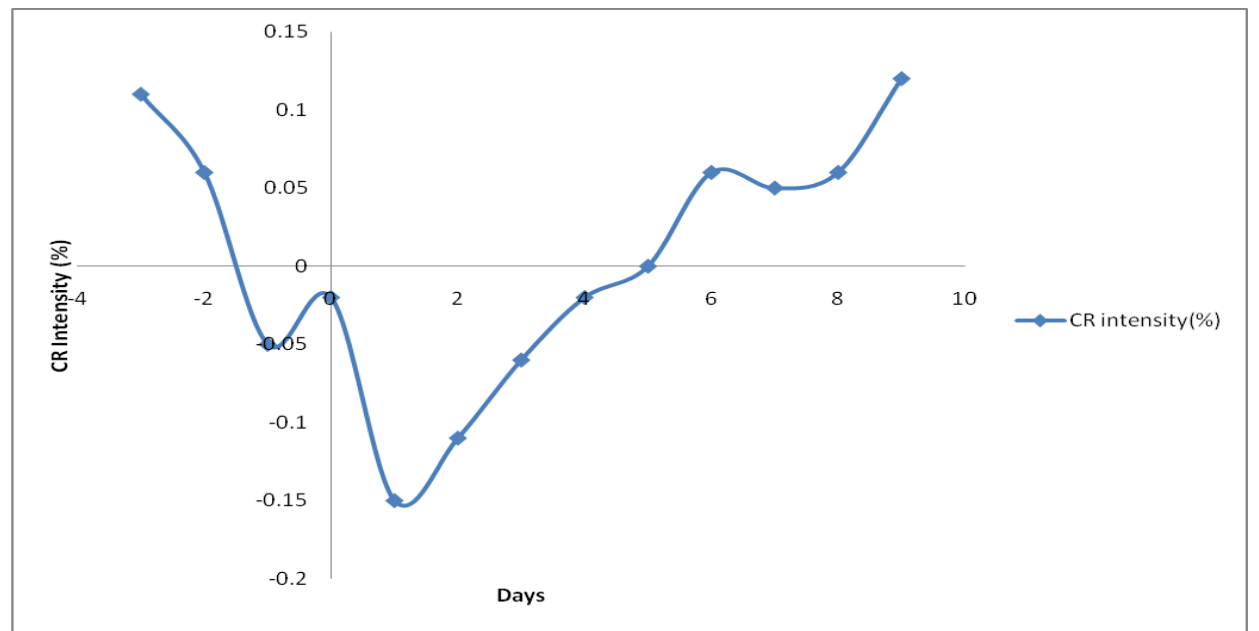

Fig 1(b): Superposed epoch plot of Oulu CR Intensity (\%) for medium high speed solar wind streams during 1996.

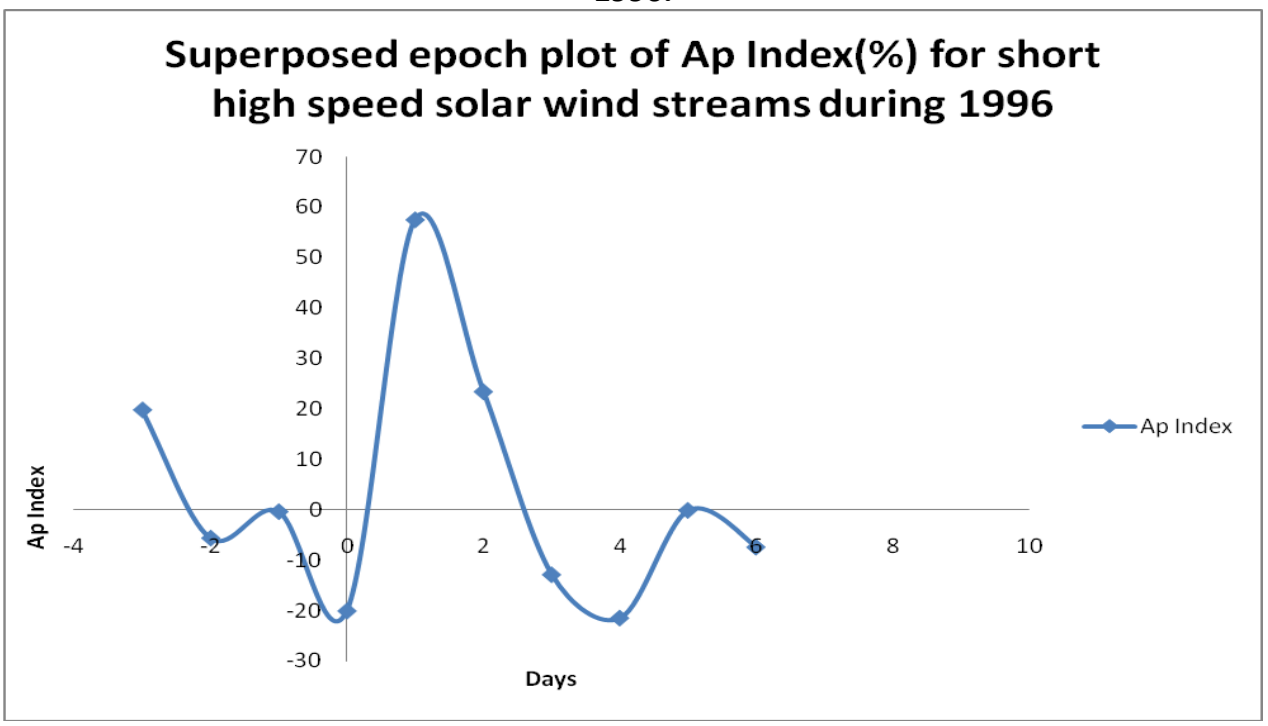

Fig 1(c): Superposed epoch plot of Ap Index (\%) for short high speed solar wind streams during 1996.

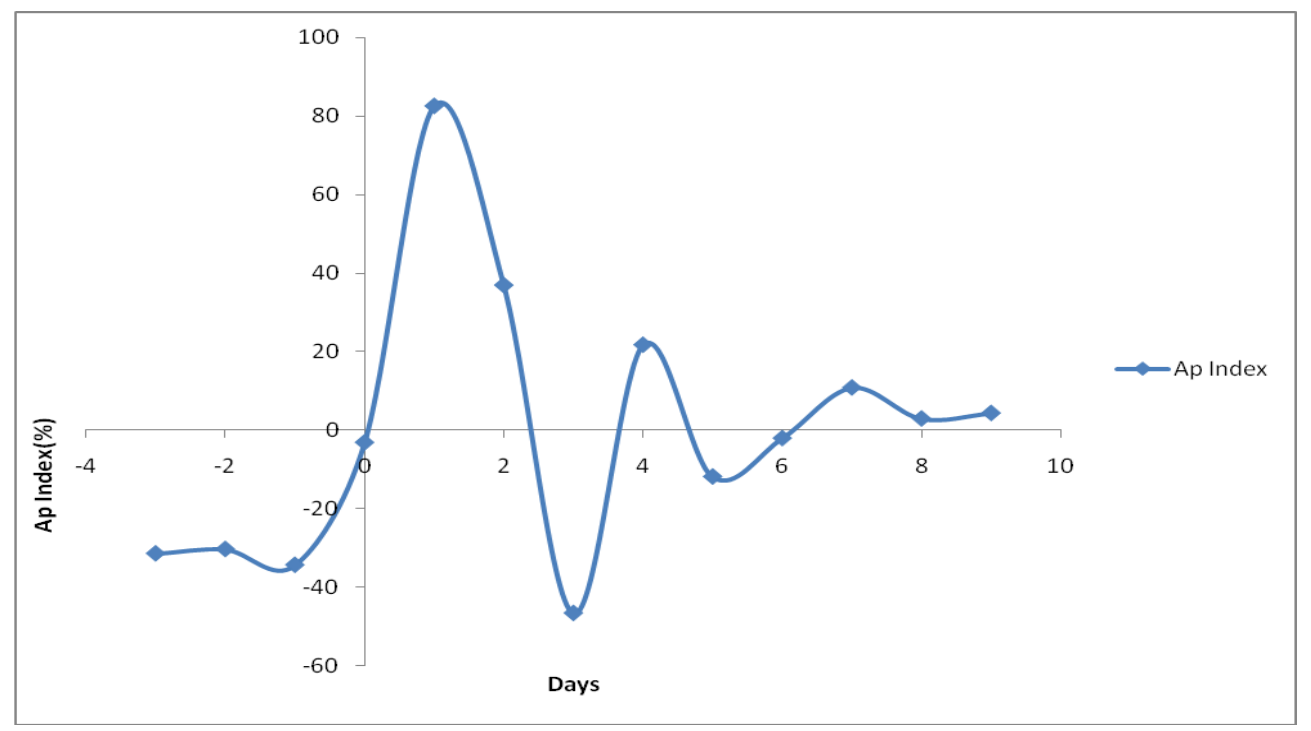

Fig 1(d): Superposed epoch plot of Ap index (\%) for medium high speed solar wind streams during 1996. 


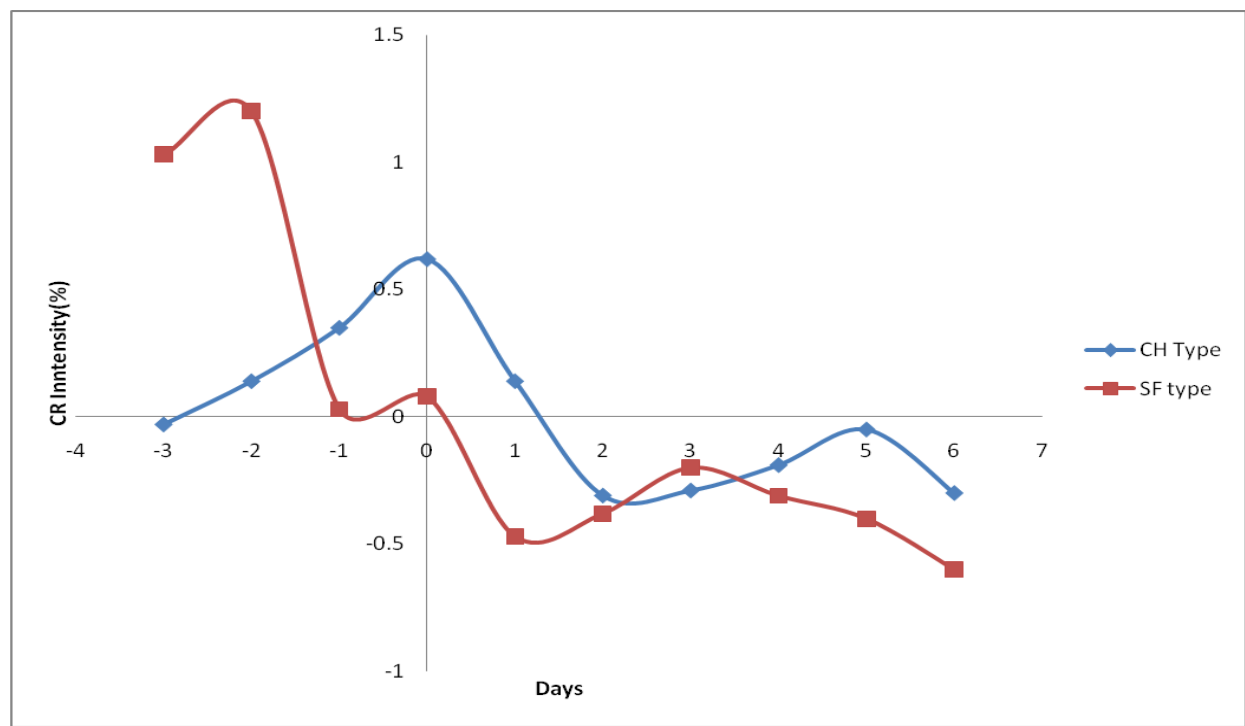

Fig 2(a): Superposed epoch plot of Oulu CR Intensity(\%) for short high speed solar wind streams during 2000.

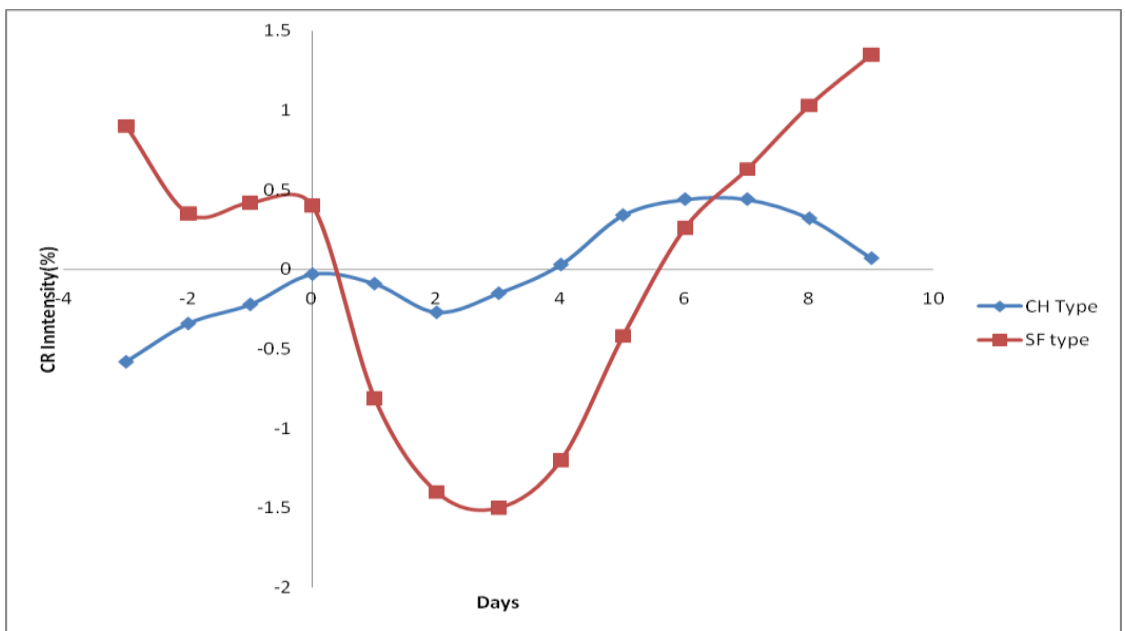

Fig. 2(b): Superposed epoch plots of Oulu CR Intensity (\%) for medium high speed solar wind streams during 2000.

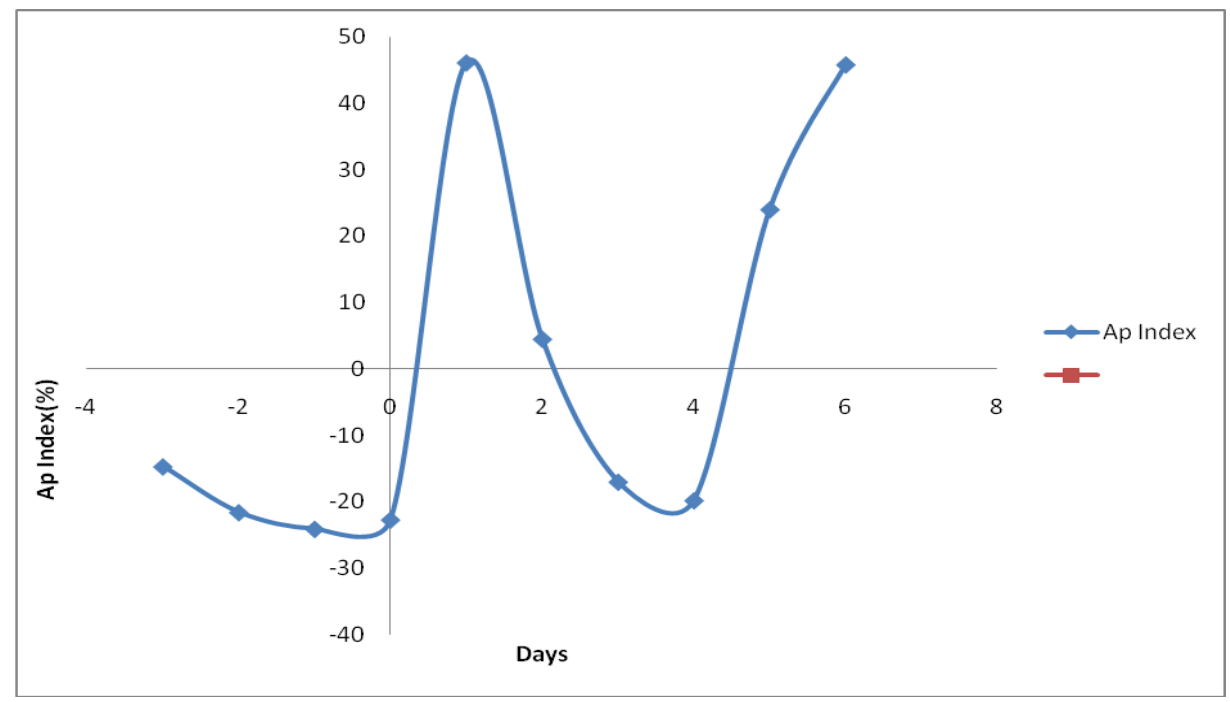

Fig 2(c): Superposed epoch plot of Ap Index(\%) for short high speed solar wind streams during 2000. 


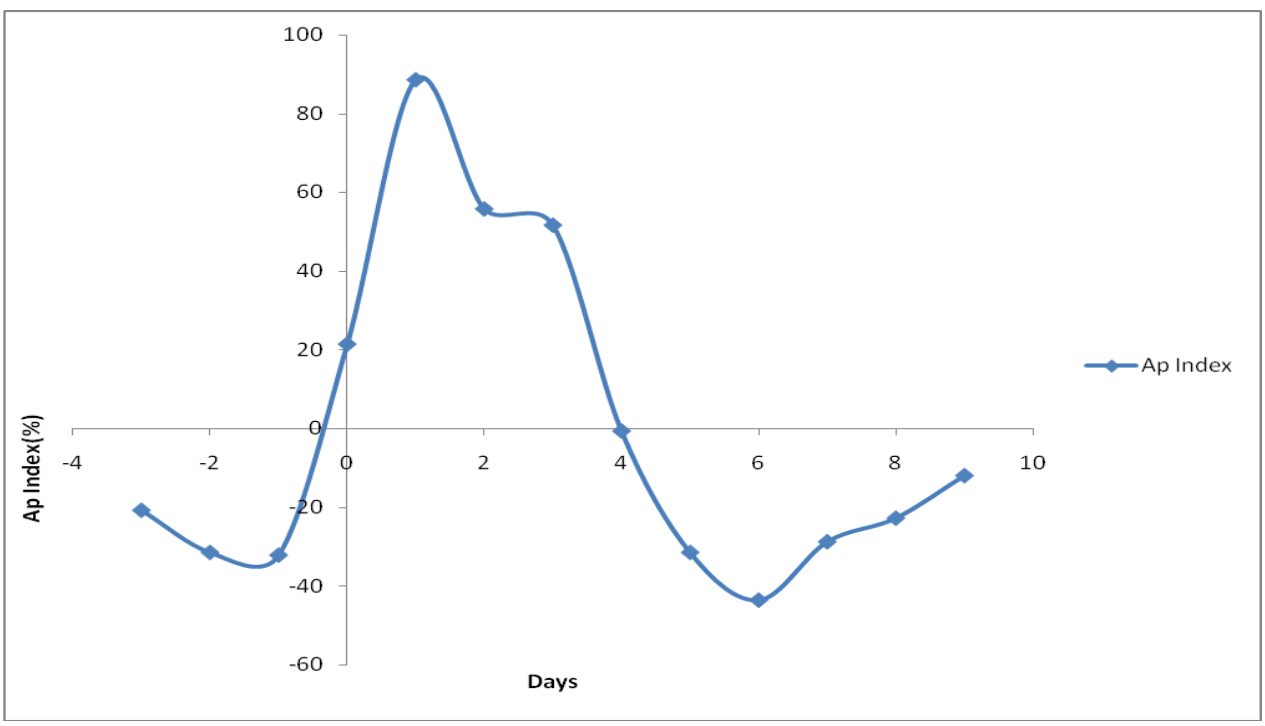

Fig 2(d): Superposed epoch plot of Ap index (\%) for medium high speed solar wind streams during 2000.

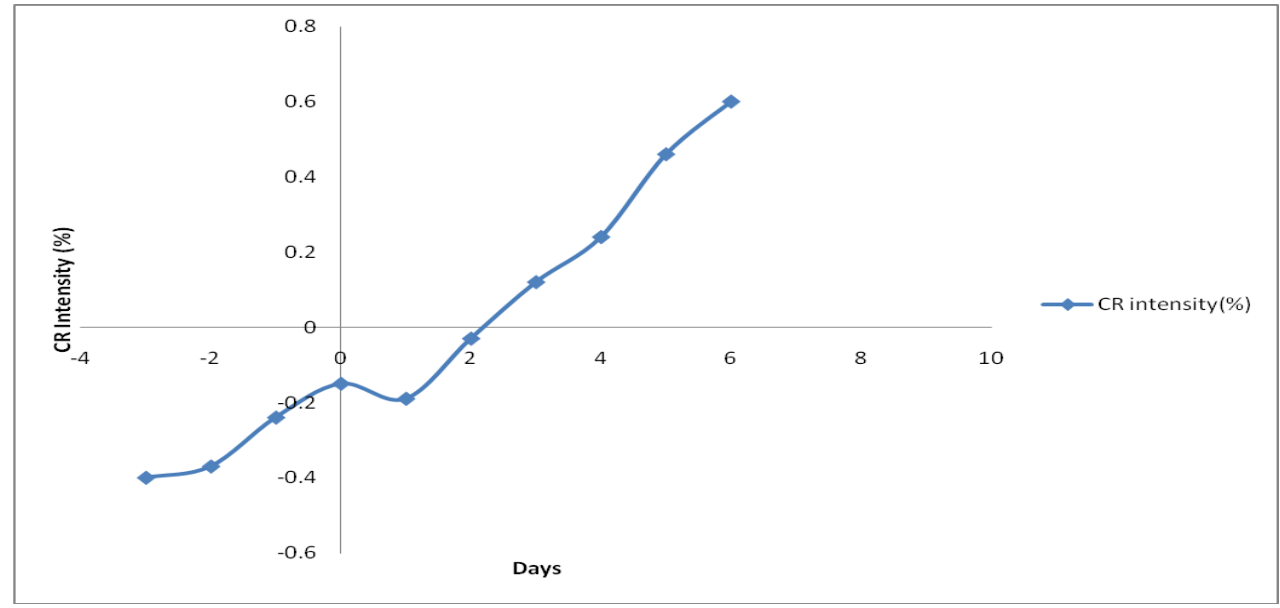

Fig 3(a): Superposed epoch plot of Oulu CR Intensity (\%) for short high speed solar wind streams during 2008.

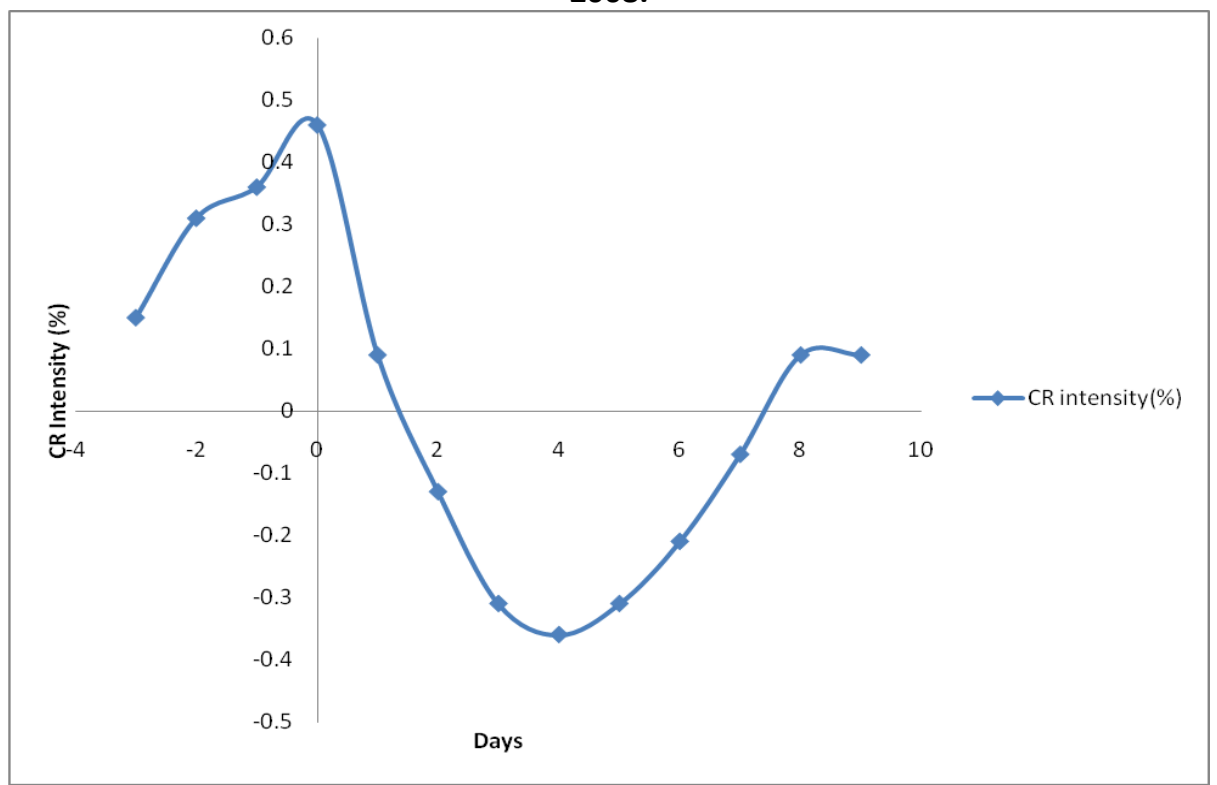

Fig 3(b): Superposed epoch plot of Oulu CR Intensity (\%) for medium high speed solar wind streams during 2008. 


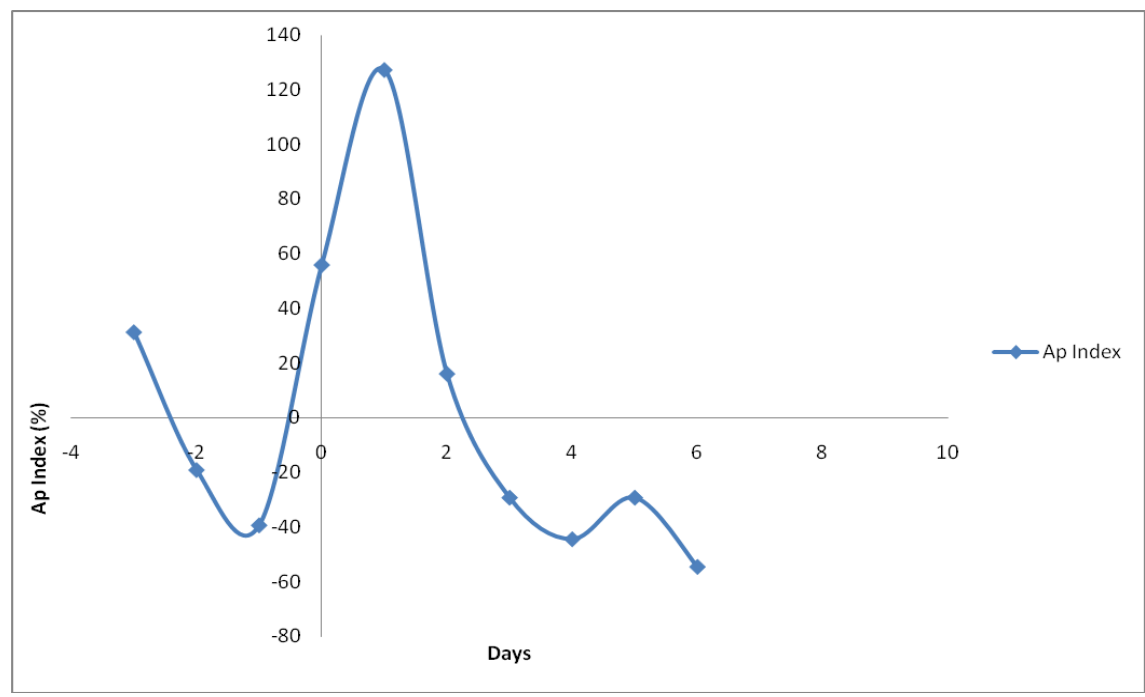

Fig 3(c): Superposed epoch plot of Ap Index(\%) for short high speed solar wind streams during 2008.

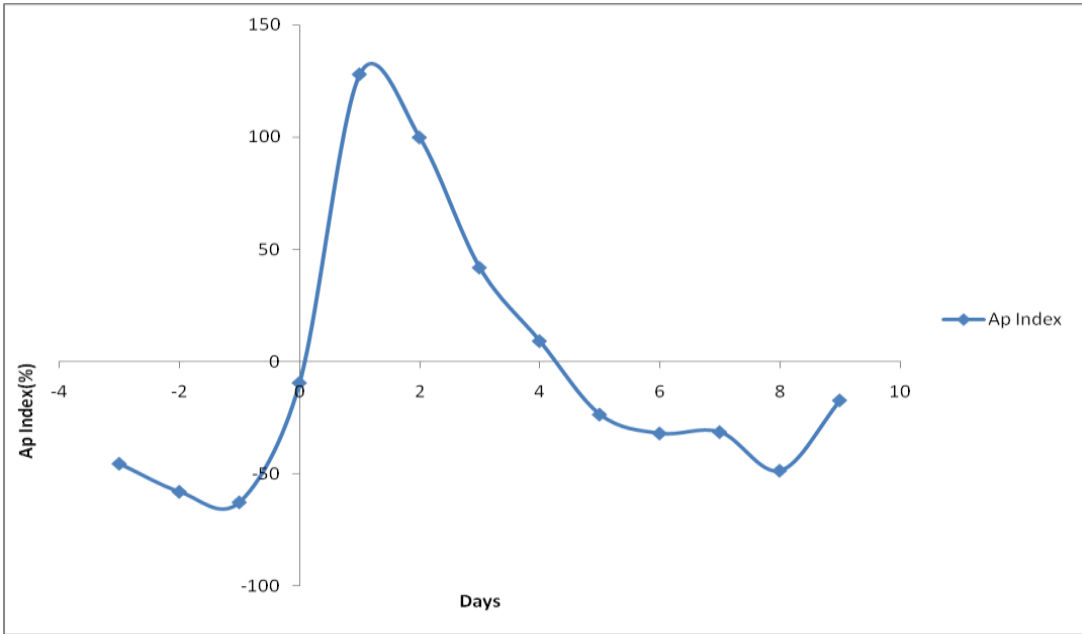

Fig 3(d): Superposed epoch plot of Ap index (\%) for medium high speed solar wind streams during 2008. 